\title{
ISOPHOT Surveys and the Extragalactic Background
}

\author{
D. Lemke, P. Ábrahám, M. Haas, P. Héraudeau, S. Hotzel, C. Kiss, U. \\ Klaas, O. Krause, C. Leinert, K. Meisenheimer, M. Stickel, L. V. Tóth \\ Max-Planck-Institut für Astronomie, Königstuhl 17, D-69117 \\ Heidelberg, Germany
}

M. Juvela, K. Mattila

University of Helsinki, Observatory, Helsinki, Finland
R. Chini, S. A. H. Müller
Ruhr-Universität Bochum, Astronomisches Institut, D-44780 Bochum, Germany

\author{
M. J. D. Linden-Vørnle, H. U. Nørgaard-Nielsen \\ Niels Bohr Institute for Astronomy, Physics and Geophysics, \\ Astronomical Observatory, Copenhagen, Denmark
}

\begin{abstract}
The higher spatial resolution and sensitivity of ISO allowed several extragalactic surveys to be extended to greater depth than obtained with IRAS. With the extended wavelength range deep surveys were performed for the first time at wavelengths up to $\sim 200 \mu \mathrm{m}$. They favour galaxy models with strong evolution. With ISO's new capabilities the spectral energy distributions of larger samples of ULIRGs in the local universe and those of quasars and radio galaxies were determined. These data are applicable as templates to the more distant universe. Foreground components from zodiacal light and cirrus to the intracluster dust emission were studied in connection with their separation from the extragalactic background radiation.
\end{abstract}

\section{Introduction}

Four types of far infrared surveys were carried out with the ISOPHOT instrument (Lemke et al. 1996):

(1) At $170 \mu \mathrm{m}$ the largest sky coverage but the shallowest depth was obtained with the "Serendipity Survey" performed during slews of the ISO satellite.

(2) Several extragalactic surveys served the goal of obtaining complete spectral energy distributions from 10 to $200 \mu \mathrm{m}$ for different classes of galaxies.

(3) Deep surveys at 90 to $180 \mu \mathrm{m}$ searched for the as yet unresolved galaxies of the Extragalactic Background Light (EBL).

(4) In order to separate foreground components from the residual EBL the straylight, zodiacal light, cirrus and intergalactic dust emission have been studied. 

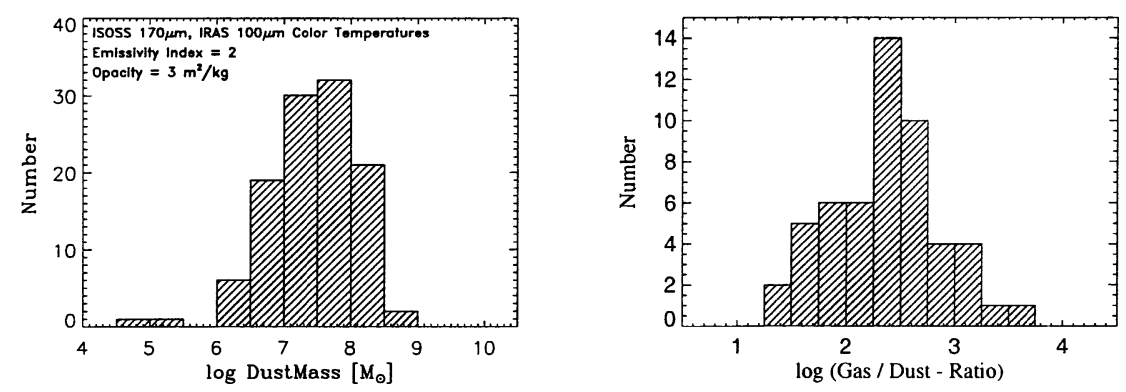

Figure 1. Histogram of dust masses determined for Serendipity Survey galaxies (left). Histogram of gas/dust ratio as determined for Serendipity Survey galaxies (right).

\section{Serendipity Survey}

With a total of $\sim 150000^{\circ}$ slew length, about $15 \%$ of the sky was covered. The slew speed of $7^{\circ} \mathrm{min}^{-1}$ allowed $\sim 0.8 \mathrm{~s}$ integration time on a point source resulting in a detection limit of $\sim 1 \mathrm{Jy}$. The first major catalogue issued contains exclusively galaxies detected by all 4 pixels of the C200 camera with a $\mathrm{S} / \mathrm{N}>5$ in faint cirrus regions at galactic latitudes $|b|>15^{\circ}$. The 115 entries represent the largest galaxy catalogue at $170 \mu \mathrm{m}$ today (Stickel et al. 2000). Combined with the relevant 60 and $100 \mu \mathrm{m}$ fluxes of the IRAS catalogue, FIR colour temperatures and masses of the very cold dust component $(\sim 17 \mathrm{~K})$ were derived. Fig. 1 shows that the dust masses have increased considerably as compared to IRAS results and correspondingly the gas-to-dust-ratios came down to a narrow range around the galactic value of $\sim 160$.

At present the Serendipity Survey work is being extended in 3 directions:

(1) The extragalactic data base will increase to hundreds of galaxies by relaxing the as yet strong selection criteria (allowing lower galactic latitudes, detection in only 3 pixels, etc.).

(2) Ground-based follow-up observations to study morphology and spectra of the bright FIR galaxies; see an example in Fig. 2.

(3) Very cold knots in the galactic foreground will be catalogued; results on the test region Chamaeleon are described by Tóth et al. (2000).

\section{ULIRG and Quasar Surveys}

The spectral energy distributions of more than 40 ultraluminous IR galaxies were measured over the full wavelength range $10-200 \mu \mathrm{m}$. The extension to $200 \mu \mathrm{m}$ as compared to the $100 \mu \mathrm{m}$ limit of IRAS has not significantly increased the luminosity derived for these objects, since the peak of the SEDs is already near $100 \mu \mathrm{m}$. In most cases the suspected AGN powered objects show a hotter dust component in the mid IR. A few examples are shown in Fig. 3; details are given by Klaas et al. (2001). Although ULIRGs of the local universe are studied here, detailed knowledge of their spectra now obtained and the physics involved can be applied as a template to the more distant universe. 

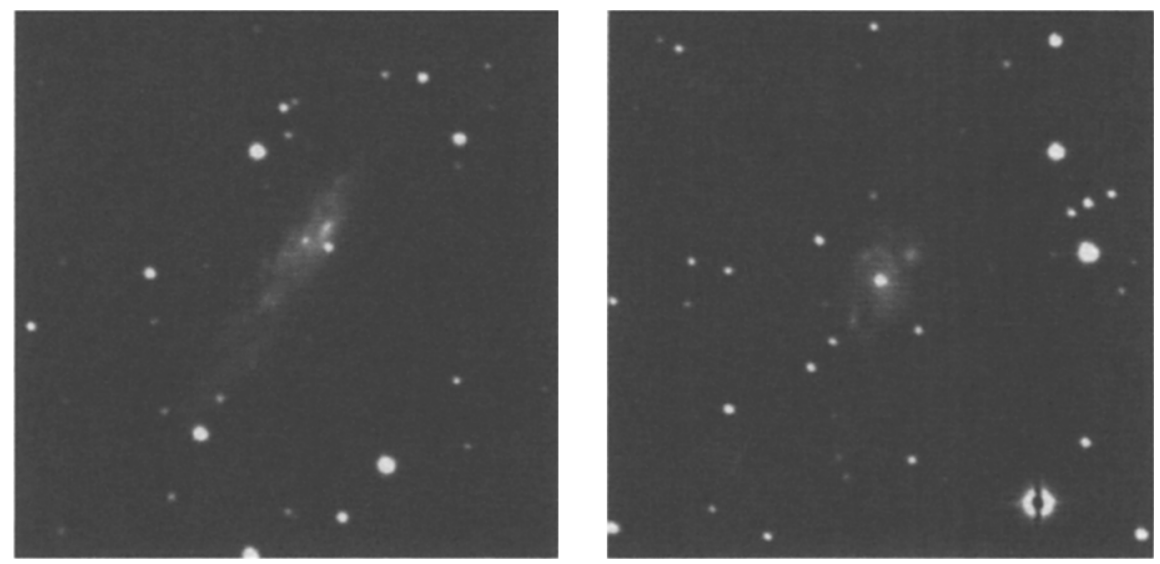

Figure 2. Optical identifications of galaxies detected in the $170 \mu \mathrm{m}$ Serendipity survey show a high percentage of disturbed morphology or close pairs.
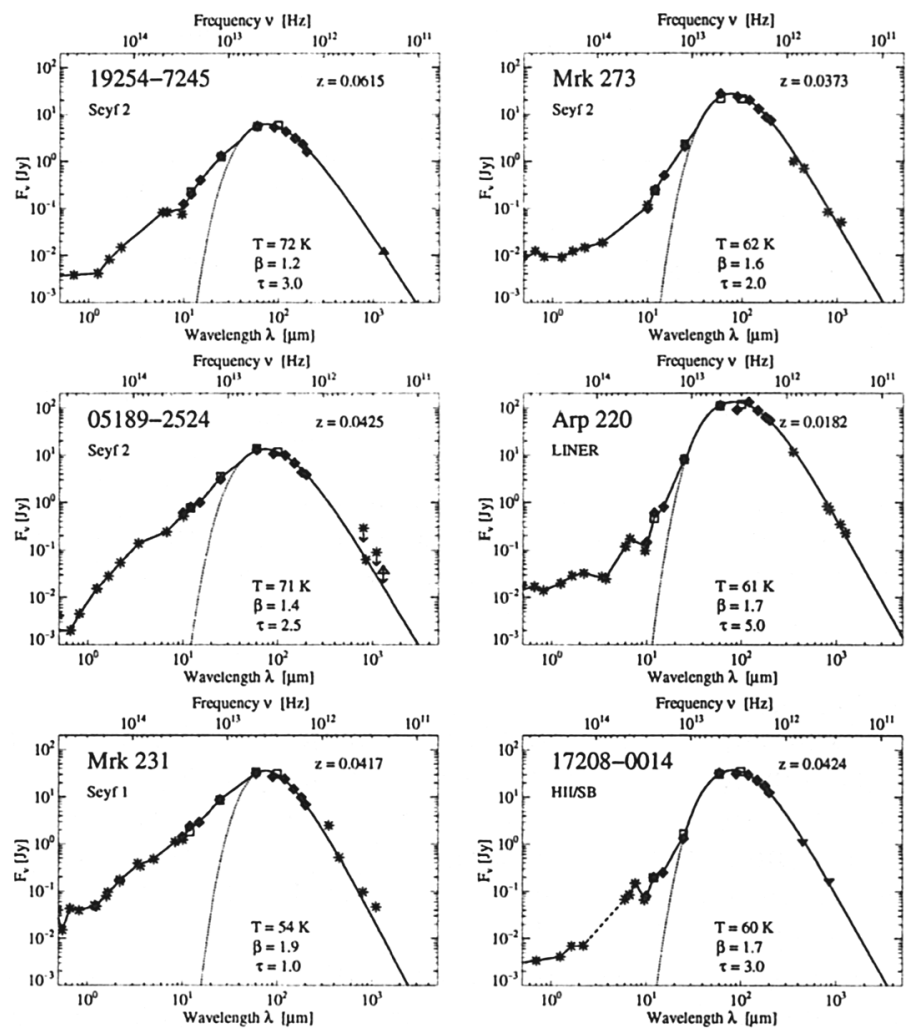

Figure 3. Examples of ULIRG spectra. The left column contains objects with higher MIR fluxes indicating powering by an AGN. 


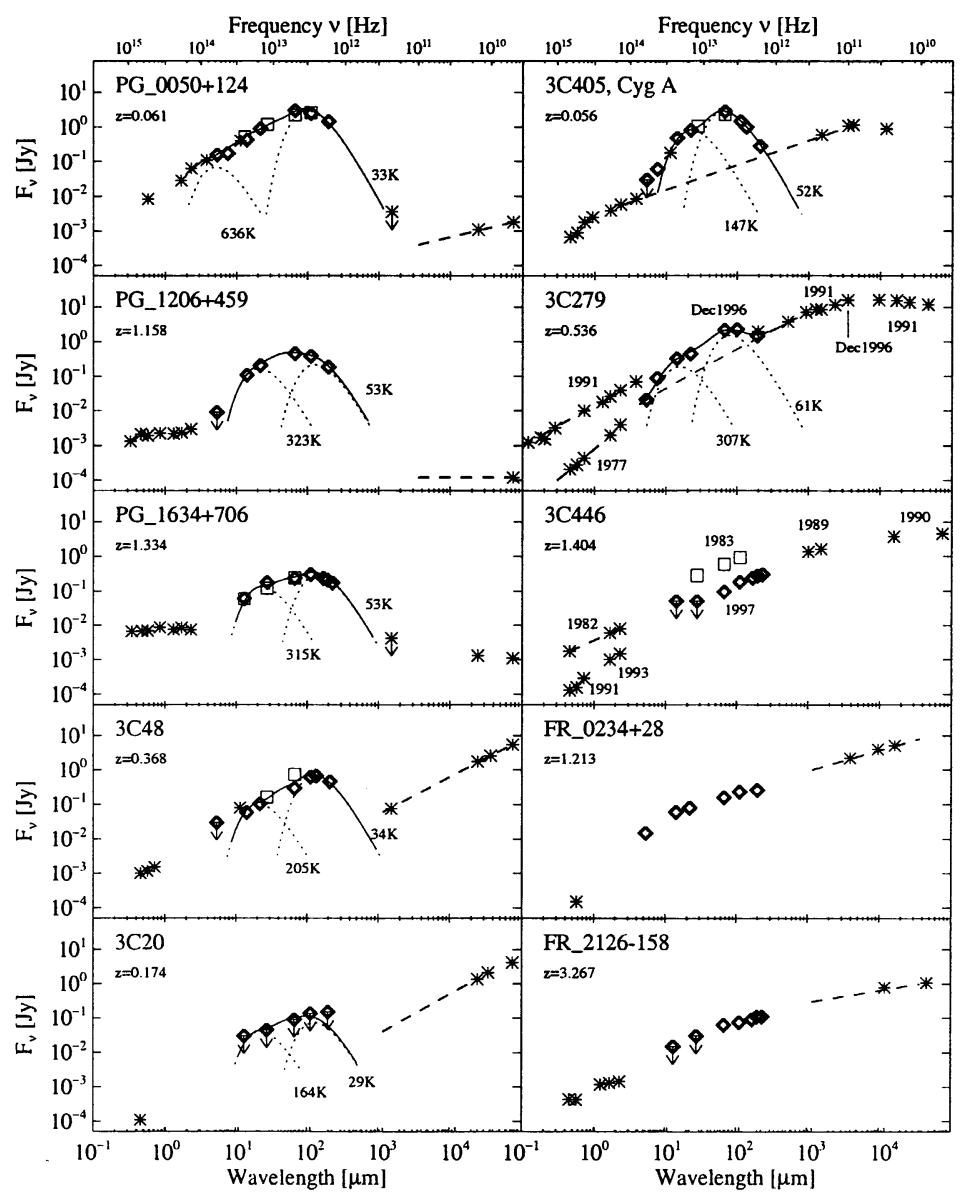

Figure 4. Spectra of quasars and radio galaxies: The spectra show similar dust bumps in the FIR, as long as the beamed synchrotron emission is not too strong to outshine the dust emission.

In our quasar survey we studied more than 50 objects with FIR fluxes $>0.1 \mathrm{Jy}$. Dust "bumps" are obvious in most objects. They can be fitted with dust colour temperatures in the 30 to $50 \mathrm{~K}$ range for the coldest dust component and, with the FIR luminosity now determined, lead to dust masses in the $10^{8}$ to $10^{6} \mathrm{M}_{\odot}$ range as are typical, respectively, for spiral and elliptical galaxies (Haas et al. 1998; Haas et al. 2000); see Fig. 4.

Quasars and radio galaxies with similar radio luminosities and redshifts selected pairwise allowed the unified models for these active galaxies to be checked. In the far infrared they should appear similar due to the isotropic dust emission and the negligible extinction. Only in objects seen pole-on does the Doppler boosted synchrotron emission wipe out the dust emission (Fig. 5). Meisenheimer et al. (2001) confirm the predictions of the unified model with their sample. 

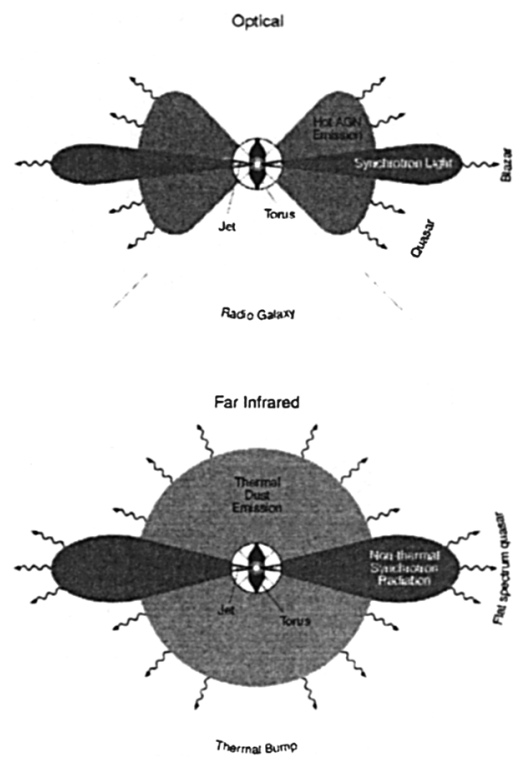

Figure 5. Schematic illustration of the unified scheme of quasars and radio galaxies.

\section{Deep Surveys}

The largest fields mapped at $170 \mu \mathrm{m}$ with ISOPHOT were the FIRBACK regions (Dole et al. 2000). This redundantly mapped survey revealed numerous sources suspected of being galaxies at intermediate $(\mathrm{z} \sim 0.5)$ redshifts. Several deep $170 \mu \mathrm{m}$ surveys were also performed by Japanese groups (Okuda 2000). Follow-up observations by radio interferometry (to determine the position) and by optical spectroscopy confirmed the nature of the brighter $170 \mu \mathrm{m}$ sources as redshifted galaxies (Sanders, private communication). Several smaller fields with low cirrus foreground (as checked by $100 \mu \mathrm{m}$ surface brightness and HI column density) were surveyed at three wavelengths between 90 and $180 \mu \mathrm{m}$ (Juvela et al. 2000). Multiwavelength data were used to confirm the source detections, and the IR colours of the objects were used to discriminate cirrus knots from galaxies. In the selected area SA57, a deep survey at $90 \mu \mathrm{m}$ was performed (Linden-Vørnle et al. 2000). Again at $90 \mu \mathrm{m}$, about 13 square degrees were mapped in the ELAIS project (Efstathiou et al. 2000). The results are plotted in $\log \mathrm{N}-\log \mathrm{S}$ diagrams and are compared with current galaxy evolution models (Fig. 6). Although different regions were scanned and different point source identification methods were applied by the 5 groups shown in Fig. 6, the results agree quite well. At $90 \mu \mathrm{m}$ no clear preference for any model can be established. However, at 150 and $180 \mu \mathrm{m}$ the source counts favour models with evolution, e.g., with a higher percentage of more luminous galaxies at higher redshifts. 
Observations $\longmapsto$ Juvela, Mattila \& Lemke 2000

O Puget, Lagache, Clements et al 1999

$\triangle$ Kawara, Sato, Matsuhara et al 1998

$\star \quad$ Linden-Vørnle, Nørgaard-Nielsen et al 2000

$\diamond$ Oliver, Serjeant, Efstathiou et al 2000

Models — Franceschini, Andreani \& Danese 1998

--- Guiderdoni, Hivon, Bouchet et al 1998

.... $90 \mu \mathrm{m}$ Guiderdoni et al 1998

150, $180 \mu \mathrm{m}$ Franceschini et al 1998

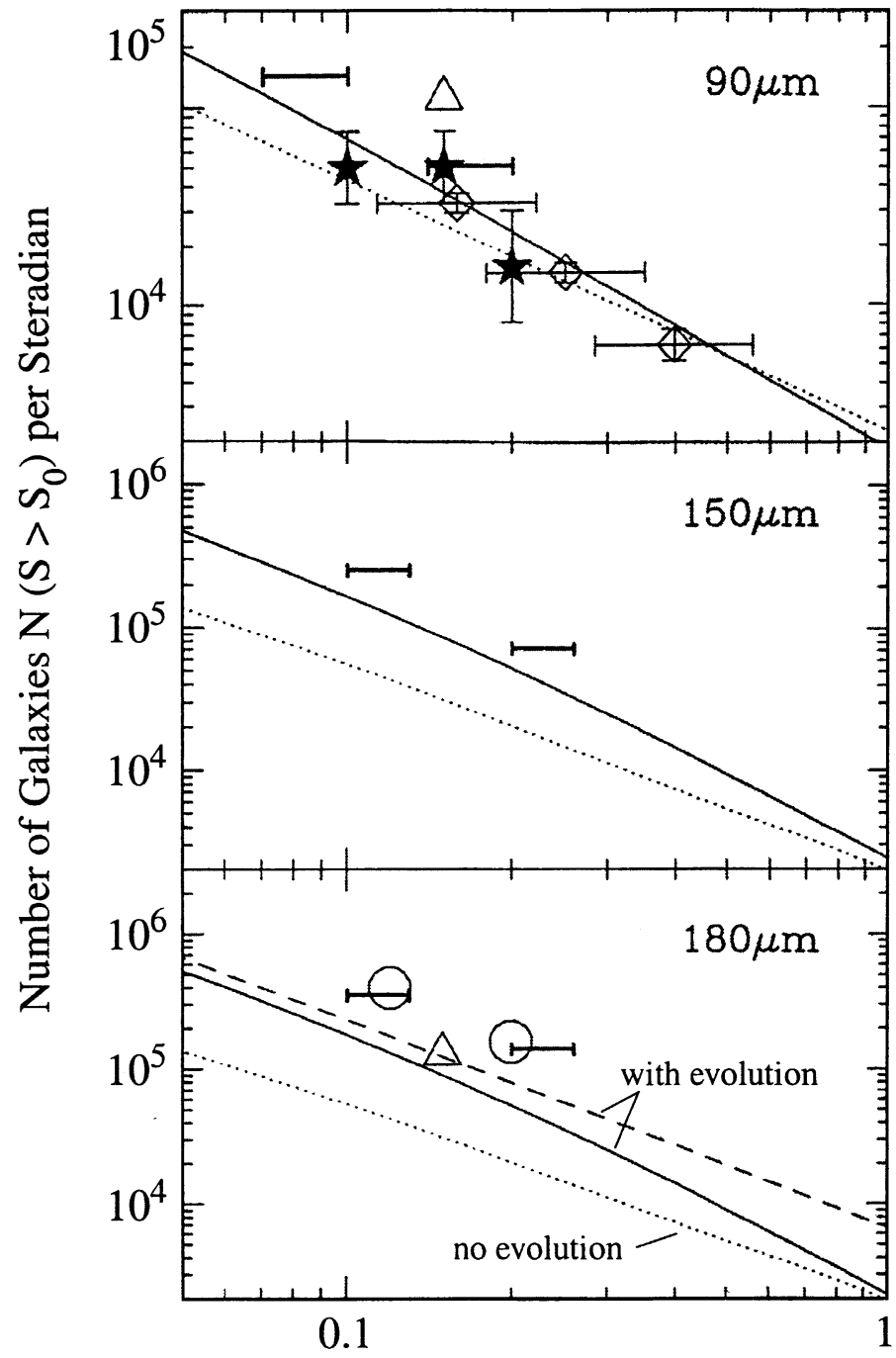

Flux $\mathrm{S}_{0}[\mathrm{Jy}]$

Figure 6. Galaxy counts compared to evolutionary models of various authors, see top of figure. 


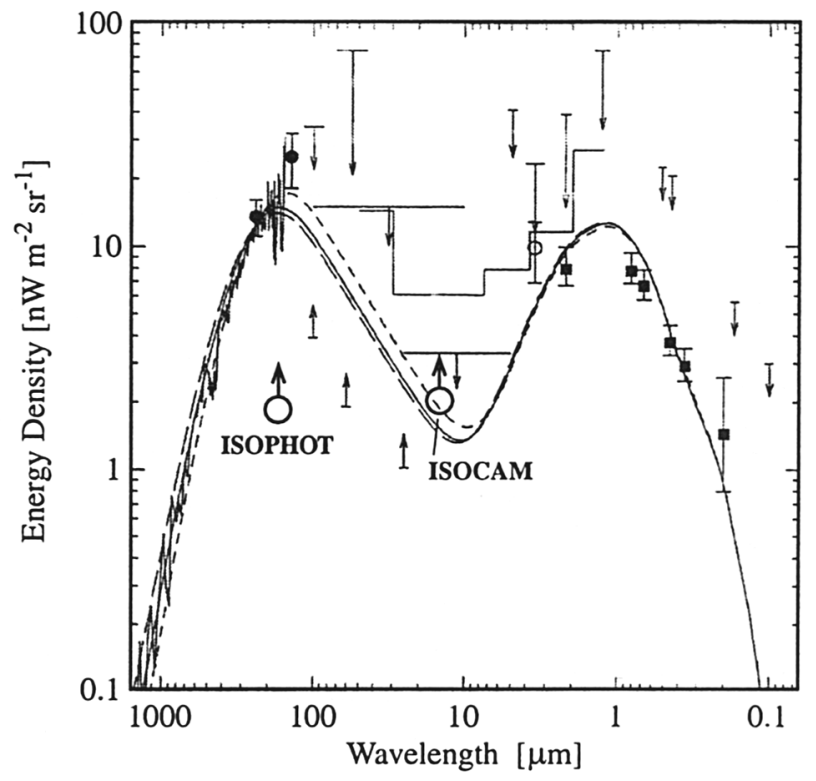

Figure 7. The integrated flux of all $170 \mu \mathrm{m}$ sources detected in ISO's deep field can account for $\sim 15 \%$ of the EBL as determined by COBE (Pei, Fall, \& Hauser 1999).

Assuming that the sources detected are indeed galaxies, then their contribution to the total EBL as measured by COBE can be estimated as about $15 \%$ at $170 \mu \mathrm{m}$ (see Fig. 7). All surveys mentioned above achieved a depth of 50 to $100 \mathrm{mJy}$. Fainter sources were not detected as individual objects but they still contribute to the surface brightness fluctuations. This fact has been used to extend the source count predictions to lower flux densities (Lagache \& Puget 2000; Matsuhara et al. 2000). Juvela et al. (2001) carried out fluctuation analysis of ISOPHOT maps observed in the North Galactic Pole region, and, in agreement with the previous results, a tentative detection of the EBL fluctuations was obtained at a level of $\gtrsim 0.05 \mathrm{MJy} \mathrm{sr}^{-1}$.

\section{Extended Foreground Emission}

\subsection{Zodiacal Light}

A network of 3 arcmin wide target points selected for "no stars, no cirrus" was established for the sky areas accessible to ISO (solar elongations $60^{\circ}<\epsilon<$ $120^{\circ}$ ). Spectral energy distributions over the range 5 to $200 \mu \mathrm{m}$ were repeatedly obtained from these target points during the mission. These data are used to calibrate the COBE zodiacal light model in the photometric system of ISOPHOT and to derive the relevant zodiacal light correction for all EBL maps. Further refinement of the zodiacal light models will be based on MIR spectra obtained with ISOPHOT-S and resulting in a clear colour temperature distribution over 

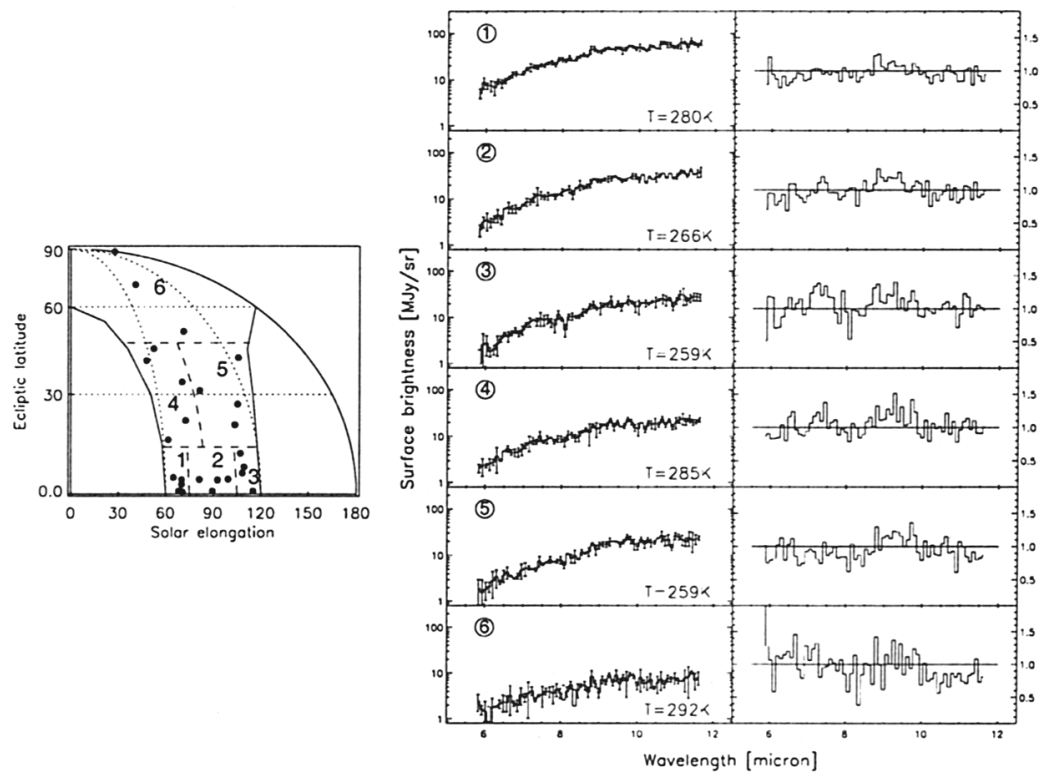

Figure 8. The MIR Spectra of the zodiacal light shown in the column of six central panels are featureless (no silicates) and show different dust colour temperatures in different sky regions (1 to 6) indicated in the map on the left, and labelled with ecliptic coordinates with the Sun at the centre. The histograms in the six panels on the right represent the residuals when the corresponding curves in the central panels are divided by blackbody fluxes at the indicated temperatures.

the zodiacal cloud; for details see Ábrahám et al. (2000) and Fig. 8. We estimate that the surface brightness of the zodiacal light at $200 \mu \mathrm{m}$ can be determined with an accuracy of $\pm 0.2 \mathrm{MJy} \mathrm{sr}^{-1}$.

\subsection{Cirrus}

The small scale structure of the cirrus can be characterized by the fluctuation power as a function of spatial frequency. The power law found by Gautier et al. (1992) based on IRAS results has been extended to higher spatial frequencies at $90 \mu \mathrm{m}$ by ISOPHOT (Herbstmeier et al. 1998). This relation was also studied for the first time at $170 \mu \mathrm{m}$ and similar exponents $\alpha \sim-1.8$ to -3.6 were found in three regions. With access to the full ISO archive we have studied more than 40 regions by a similar Fourier analysis. There is an indication that the exponent $\alpha$ can vary in a range from $-5<\alpha<0$; examples are given in Fig. 9 (Kiss et al. 2001). In preliminary checks we have not found any correlation of $\alpha$ with the type of region (thin cirrus, molecular clouds, ...). However, a correlation with the average brightness of the region is indicated. Studies assuming that a universal value of $\alpha \sim-3$ exists and deriving residual cirrus contribution or background fluctuations in fields where the Fourier analysis of the cirrus fluctuation has not been performed, should be considered with care. We estimate that the residual 

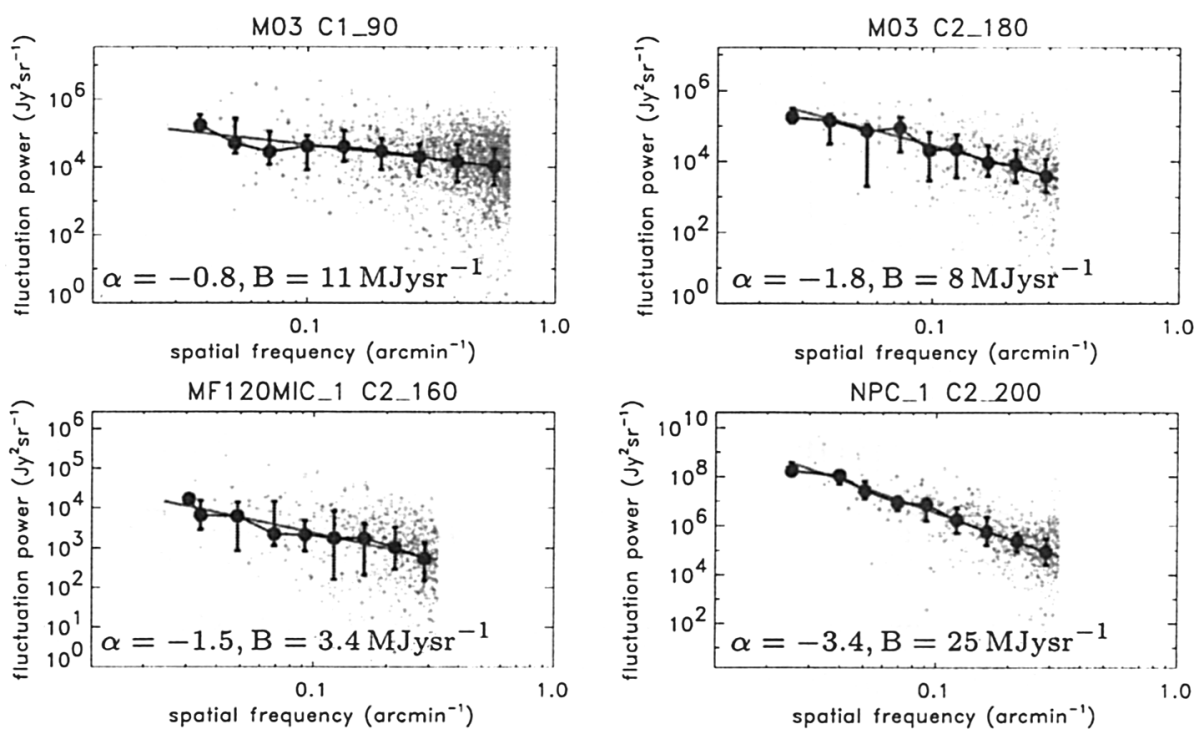

Figure 9. Structures of the galactic cirrus are characterized by Fourier analysis resulting in a power law $P=P_{0} \cdot\left(d / d_{0}\right)^{\alpha}$, where $\mathrm{d}$ is the separation between two positions in the field under investigation and $d_{0}$ is a reference length. The fluctuation power $\mathrm{P}$ depends via different spectral indices $\alpha$ on the spatial frequency. B is the average surface brightness of the cirrus region.

cirrus brightness in the ISO target fields studied with a spatial resolution of $\sim 1.5$ arcmin can be determined to $\pm 0.2 \mathrm{MJy} \mathrm{sr}^{-1}$ at $170 \mu \mathrm{m}$.

\subsection{Intracluster Dust}

In order to determine a possible contribution of intergalactic dust emission to the EBL, several galaxy clusters have been searched for intracluster dust. This was done by scanning across the cluster in 2 bands, at 120 and $180 \mu \mathrm{m}$. The ratio of fluxes I120/I180 before zodiacal light correction is shown in Fig. 10. In the Coma cluster (Abell 1656) there is an indication of the presence of a slightly hotter $(\sim 30 \mathrm{~K})$ dust component (Stickel et al. 1998). The subtraction of the zodiacal light and the correction for Galactic foreground dust emission ( $\mathrm{T} \sim 16$ $\mathrm{K})$ is in progress. It can be expected that the intergalactic dust contribution to the EBL is $<0.1 \mathrm{MJysr}^{-1}$ (Stickel et al. 2001).

\subsection{Straylight}

The straylight contribution of the bright sources Sun and Moon close to their avoidance angles could be studied during solar eclipses occurring in ISO's extended mission. The straylight at 25 and $170 \mu \mathrm{m}$ was found to be $<0.05 \mathrm{MJy} \mathrm{sr}^{-1}$. The Earth straylight was studied under the extreme case that the Earth fully illuminates the inner side of the sunshade. Its contribution was $<0.1 \mathrm{MJy} \mathrm{sr}^{-1}$. 

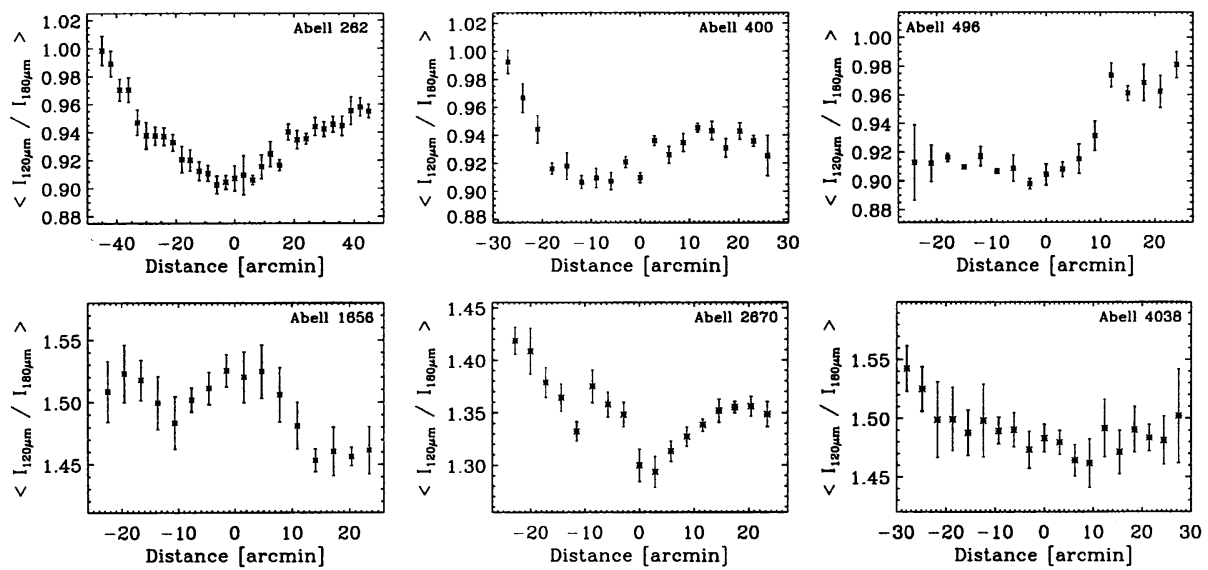

Figure 10. The flux ratio I120/I180 of scans across several galaxy clusters. Raw data before zodiacal light subtraction are plotted.

Because EBL studies were never performed under these extreme conditions, we conclude that straylight from Sun, Moon and Earth are negligible. For details see Klaas et al. (1998) and Kranz (1998).

\section{Towards an EBL Determination}

While there is already a good handle on the foreground corrections for the 150 to $200 \mu \mathrm{m}$ range, the major obstacle to determine the residual background is at present the uncertainty in the photometric calibration at these extremely low fluxes. For reasons of safety, ISO as an observatory was not equipped with a cold shutter allowing the "zero point" in the calibration scale to be determined. This is presently studied by (1) determination of the dark current along the orbit, (2) by correcting for non-linearities of the detector responsitivity at low flux levels and (3) by determining a potential instrumental straylight caused by the sky near the target. A sufficient data base to address the latter subject is available from "near field" straylight measurements performed with $20 \mathrm{arcmin}$ long scans across bright targets (stars, Saturn) and in the $1^{\circ}$ environment of Saturn from the $170 \mu \mathrm{m}$ Serendipity Survey. Contrary to the pioneering COBE measurement, where the dominating uncertainty is due to the interstellar matter contribution in the large $\left(0.7^{\circ}\right) \mathrm{FOV}$ (Hauser, this conference), we expect the ISO measurements to be limited by the zero point uncertainty. Although considerable efforts are still needed to estimate the EBL from the ISO data base, the prospects for an independent determination are realistic.

Acknowledgments. Based on observations with ISO, an ESA project with instruments funded by ESA Member States (especially the PI countries: France, Germany, the Netherlands and the United Kingdom) and with the participation of ISAS and NASA. The ISOPHOT project was funded by the Deutsche Agentur für Raumfahrtangelegenheiten DARA, the Max-Planck-Gesellschaft, the Danish, 


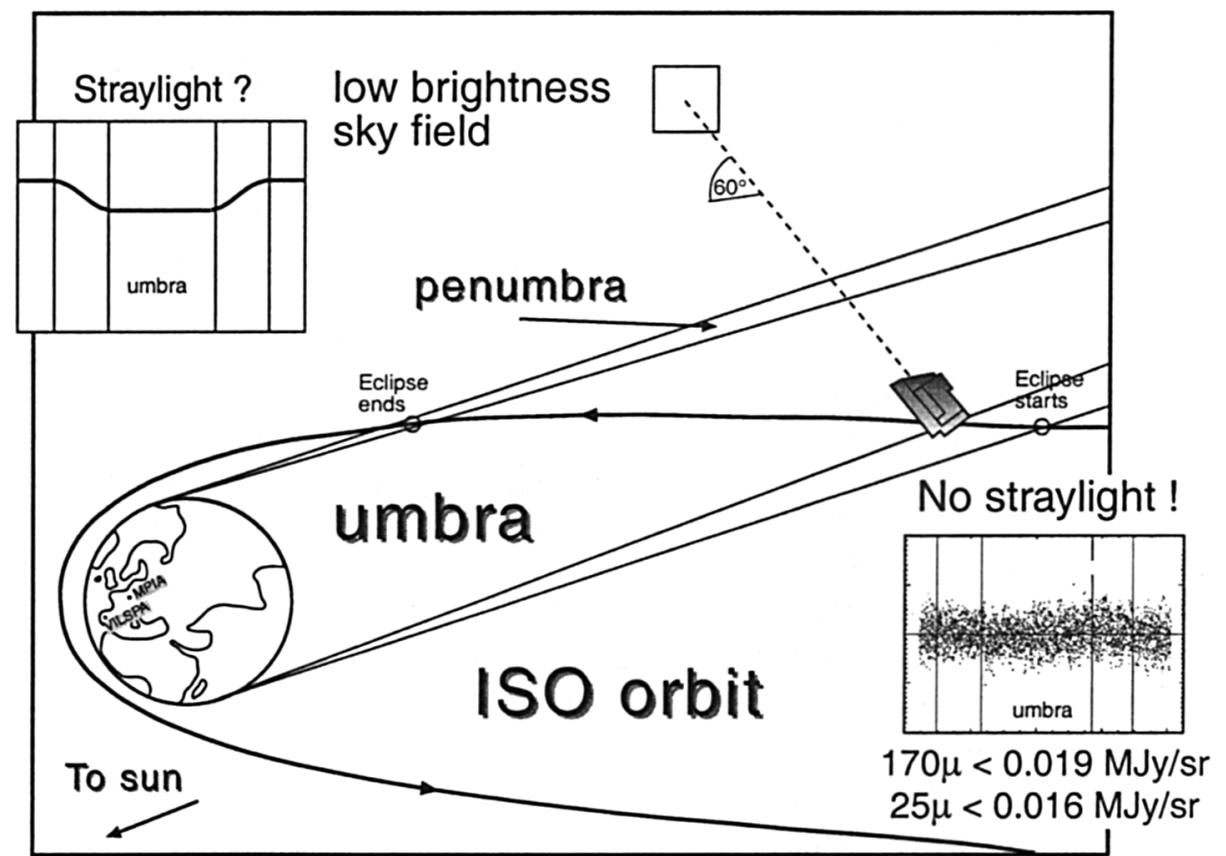

Figure 11. Sun straylight measurements were performed by observation of a dark sky field while passing the Sun's shadow. The sky signal remained constant, indicating negligible straylight from the Sun. 
British and Spanish Space Agencies and several European and American institutes. The ISOPHOT Data Centre is supported by Deutsches Zentrum für Luftund Raumfahrt e.V. (DLR) with funds of Bundesministerium für Bildung und Forschung, grant no. 50 QI 98013 . The authors are responsible for the contents of this paper.

\section{References}

Ábrahám, P., Leinert, C., \& Lemke, D. 2001, in Proc. IAU Colloquium 181 / COSPAR Colloquium 11, Dust in the Solar System and Other Planetary Systems, ed. S. F. Green et al., in press

Dole, H., et al. 2000, in Lecture Notes in Physics, 548, Ringberg Workshop Proceedings, ISO Surveys of a Dusty Universe, eds. D. Lemke, M. Stickel \& K. Wilke (Heidelberg: Springer), 54

Efstathiou, A., et al. 2000, MNRAS, 319, 1169

Francescini, A., Andreani, P., \& Danese, L. 1998, MNRAS, 296, 709

Gautier, T. N. III, Boulanger, F., Perault, M., \& Puget, J.-L. 1992, AJ, 103, 1313

Guiderdoni, B., Hivon, E., Bouchet, F., \& Maffei, B. 1998, MNRAS, 295, 877

Haas, M., et al. 1998, ApJ, 503, L 109

Haas, M., et al. 2000, A\&A, 354, 453

Hauser, M. G. 2000, in Proc. IAU Symposium 204, The Extragalactic Infrared Background and its Cosmological Implications, ed. M. Harwit \& M. G. Hauser (San Francisco: ASP)

Herbstmeier, U., et al. 1998, A\&A, 332, 739

Juvela, M., Mattila, K., \& Lemke, D. 2000, A\&A, 360, 813

Juvela, M., Mattila, K., \& Lemke, D. 2001, in preparation

Kawara, K., et al. 1998, A\&A, 336, L9

Kiss, C., et al. 2001, in preparation

Klaas, U., et al. 1998, SPIE 3354, 996

Klaas, U., et al. 2001, A\&A, submitted

Kranz, T. 1998, Measurements of Solar, Lunar and Terrestial Straylight in the ISO Telescope, diploma thesis, Universität Heidelberg (in German)

Lagache, G., \& Puget, J.-L. 2000, A\&A, 355, 17

Lemke, D., et al. 1996, A\&A, 315, L64

Linden-Vørnle, M. J. D., et al. 2000, A\&A, 359, 51

Matsuhara, H., et al. 2000, A\&A, 361, 407

Meisenheimer, et al. 2001, A\&A, in press

Okuda H. 2000, in Lecture Notes in Physics, 548, Ringberg Workshop Proceedings, ISO Surveys of a Dusty Universe, ed. D. Lemke, M. Stickel \& K. Wilke (Heidelberg: Springer), 40

Oliver, S., et al. 2000, in Lecture Notes in Physics, 548, Ringberg Workshop Proceedings, ISO Surveys of a Dusty Universe, ed. D. Lemke, M. Stickel $\&$ K. Wilke (Heidelberg: Springer), 28 
Pei, Y. C., Fall, S. M., \& Hauser, M. G. 1999, ApJ, 522, 604

Puget, J. L., et al. 1999, A\&A, 345, 29

Sanders, D. B. 2000, private communication

Stickel, M., et al. 1998, A\&A, 329, 55

Stickel, M., et al. 2000, A\&A, 359, 865

Stickel, M., Klaas, U., Lemke, D., \& Mattila, K. 2001, in preparation

Tóth, L. V., et al. 2000, A\&A, in press

\section{Discussion}

Alberto Franceschini: Have you been able to estimate preliminary galaxy counts at the level of the Serendipity Survey? They would be extremely useful for an assessment of the evolution properties of $170 \mu \mathrm{m}$ sources.

Dietrich Lemke: The detection limit of the Serendipity survey with $\sim 1 \mathrm{~s}$ integration time on the source is $\sim 1 \mathrm{Jy}$. That is one order of magnitude higher than that of dedicated deep surveys at $170 \mu \mathrm{m}$. We intend to extend the source counts to brighter sources normally not contained in deep fields. The Serendipity Survey could be a unique source for those galaxies in the $1-50$ Jy range, allowing better definition of the slope in the $\log \mathrm{N} / \log \mathrm{S}$ diagrams at the bright end.

Michael Rowan-Robinson: At what range of wavelengths do you hope to measure the background?

Lemke: The best chances are in the far infrared $(100-200 \mu \mathrm{m})$, where the zodiacal light is low and well measured and cirrus structures are avoided in the small beam. The ongoing efforts are towards establishing the calibration at the faintest level and the zero point which cannot be derived from a "cold shutter" measurement with ISO.

Michael Werner: In your $175 \mu \mathrm{m}$ Serendipity Survey did you see sources not found in the IRAS survey?

Lemke: The first large $175 \mu \mathrm{m}$ catalogue published contains galaxies that all have IRAS counterparts. Also the galactic sources published so far require the IRAS counterparts in order to determine the colour temperature of these cold dense cores. There are indeed $175 \mu \mathrm{m}$ sources not contained in the IRAS catalogues; most of them are probably cold cirrus structures. While further evaluating the large serendipity data base, we are looking for more interesting sources conspicuous at this longest wavelength.

Leonid Ozernoy: Would it be fair to say that far infrared spectra alone do not allow discrimination between AGN galaxies and luminous infrared galaxies (i.e., galaxies undergoing bursts of star formation)?

Lemke: The spectral energy distribution in the far infrared $(60-240 \mu \mathrm{m})$ is not sufficient; both types are similar. AGNs tend (depending on the viewing angle) to be brighter in the mid infrared $(5 \ldots 30 \mu \mathrm{m})$ spectral energy distribution, due to a warmer dust component. Spectra taken with higher resolution in the mid infrared allow identification of the presence of an AGN via fine structure lines of highly excited species. The strength of the PAH features is also used as a diagnostic tool (see the relevant studies of R. Genzel, D. Lutz et al. based on ISO SWS and ISOPHOT-S observations). 